Research article

\title{
Diagnostic and treatment characteristics of polycystic ovary syndrome: descriptive measurements of patient perception and awareness from 657 confidential self-reports E Scott Sills*1, Mark Perloe ${ }^{1}$, Michael J Tucker ${ }^{1}$, Carolyn R Kaplan ${ }^{1}$, Marc Georges Genton ${ }^{2}$ and Glenn L Schattman ${ }^{3}$
}

Address: ${ }^{1}$ Georgia Reproductive Specialists LLC, Atlanta, Georgia, USA, ${ }^{2}$ Department of Mathematics, Massachusetts Institute of Technology, Cambridge,Massachusetts, USA and ${ }^{3}$ Center For Reproductive Medicine \& Infertility, Weill Medical College of Cornell University, The New York-Presbyterian Hospital, New York, USA

E-mail: E Scott Sills* - dr.sills@ivf.com; Mark Perloe - mpmd@ivf.com; Michael J Tucker - mtucker@ivf.com;

Carolyn R Kaplan - ckaplan@ivf.com; Marc Georges Genton - genton@math.mit.edu; Glenn L Schattman - glschatt@mail.med.cornell.edu ${ }^{*}$ Corresponding author

Published: 22 August 200I

BMC Women's Health 200I, 1:3

This article is available from: http://www.biomedcentral.com/1472-6874/I/3

(c) 200 I Sills et al; licensee BioMed Central Ltd. Verbatim copying and redistribution of this article are permitted in any medium for any non-commercial purpose, provided this notice is preserved along with the article's original URL. For commercial use, contact info@biomedcentral.com
Received: II July 200 I

Accepted: 22 August 2001

\begin{abstract}
Background: This investigation was undertaken to describe patient perception and awareness of the polycystic ovary syndrome (PCOS), the most common cause of anovulation/oligoovulation among women of reproductive age.

Methods: Fifteen parameters were evaluated by a computer-based research instrument accessed by a large, unscreened population. Incomplete questionnaires were not entered, and responses were electronically tabulated to block duplicate submissions.

Results: From 657 participants, the majority (63\%) were between 26-34 years old; mean BMI was $30.4 \mathrm{~kg} / \mathrm{m}^{2} .343$ of $657 \mathrm{had}$ at least one pregnancy and $61 \%$ of the study group had taken fertility medicine (any type) at least once. Physicians were the most common provider of PCOS information for all study participants, irrespective of age. Patient emotions associated with the diagnosis of PCOS included "frustration" (67\%), "anxiety" (16\%), "sadness" (10\%), and "indifference" (2\%). Self-reported patient aptitude regarding PCOS was scored as high or "very aware" in $>60 \%$ of women. Respondents were also asked: "If your PCOS could be safely and effectively helped by something else besides fertility drugs or birth control pills, would that interest you?" Interest in alternative PCOS treatments was expressed by $99 \%$ of the sample $(n=648)$.

Conclusions: In our study population, most women associated negative emotions with PCOS although the self-reported knowledge level for the disorder was high. While these women regarded their obstetrician-gynecologist as integral to their PCOS education, traditional PCOS therapies based on oral contraceptives or ovulation induction agents were regarded as unsatisfactory by most women.
\end{abstract}




\section{Background}

Little is known of how patients with the polycystic ovary syndrome (PCOS) acquire information about their condition, whether they feel their level of understanding about PCOS is adequate, or what emotions are associated with this diagnosis. Some of the components of PCOS are known to be profoundly stressful, yet effective and systematic efforts to characterize the subjective awareness of this disorder have only recently been developed [1]. Mainstream treatments for PCOS have remained fairly fixed over the past 30 years, with the two principal nonsurgical approaches being oral contraceptives and clomiphene citrate. PCOS therapies based on orally-active insulin sensitizers account for limited clinical use at present, although preliminary data suggests an acceptable safety and efficacy profile for such treatment [2-4].

In this study, we wished to describe the self-reported knowledge base for PCOS in a large, unscreened patient group, as well as to identify where patient information about PCOS originates. We were especially interested to learn which emotions PCOS patients ascribe to their problem once the diagnosis is made, and how they feel about the treatments proposed for their condition. Additionally, this study sought to measure women's receptiveness to alternative treatment strategies not predicated on oral contraceptives or ovulation induction when queried in a patient-centered, confidential and anonymous manner.

\section{Methods}

A multidisciplinary team developed a 15-item research questionnaire specifically targeted to women with PCOS. Queries were structured as single-answer from multiple choice responses and data tabulated accordingly for analysis; the questionnaire was then configured electronically for internet access [see link: [http://www.obgyn.net/cfm/pcos-survey/pcos-survey.cfm] ]. Although participants were required to register via computer in order to access the questionnaire site, there was no cost to do so. When a group of volunteers $(n=12)$ pre-tested the study questionnaire, the mean time required for completion was 8 min (including registration). The PCOS questionnaire was posted in English and continuously maintained on a free public-access medical internet site (OBGYN.net; Austin, TX) throughout the month of January, 2000. The questionnaire site was identifiable and its recognition confirmed via three major internet search engines directed to the terms "PCOS" or "PCOS studies".

Participation in this open study was not restricted to current or former patients of the investigators, but was made available to all women who had been diagnosed with PCOS from any center. Participants received no compensation or acknowledgement for their reply to this study questionnaire. Incomplete questionnaires were not tabulated, and responses were electronically tracked to block duplicate submissions from the same individual. Height and weight information was received from participants either in Imperial (U.S.) or SI (metric) units, with automated metric conversion for analysis. Only investigators were permitted access to running totals of the questionnaire during the study.

Our research design was submitted to the Institutional Review Board of Atlanta Medical Center prior to questionnaire implementation. As our study required no direct patient contact or medical record review, the research protocol was judged as "no risk to human subjects"; IRB oversight was therefore offered but not required.

Chi-square/contingency table test was used to determine dependence between measured variables in the data array. $P$ values $<0.05$ were considered significant.

\section{Results}

During the study interval, 657 unique responses to the questionnaire were registered. More than $97 \%(n=638)$ of the respondents indicated that they were familiar with PCOS, while $1.9 \%$ had not been told about PCOS, and $<1 \%$ were uncertain. Approximately $61 \%$ of women regarded their level of understanding for PCOS as "very aware" ( $n=403)$, but $9.1 \%$ and $2.9 \%$ of women considered themselves to be "minimally aware" or "not aware at all" regarding PCOS, respectively. The proportion of the sample registering as ever-pregnant was $52.2 \%(n=$ 343 ), and the average number of pregnancies reported (including miscarriages) was 2.08/patient. Median [IQR $25 ; 75]$ height and weight in this study population was $165.1[160 ; 170] \mathrm{cm}$ and 86.0 [70;104] kg, respectively $\left(\right.$ median BMI $\left.=31.9 \mathrm{~kg} / \mathrm{m}^{2}\right)$.

A summary of responses stratified by age and other parameters is given in Figures 1,2,3,4,5,6,7. When these demographic data were related to the self-reported "awareness" of PCOS among these women, those subjects between age 26-34 were significantly more "aware" of PCOS than any other age group $(p=0.038)$. This age group was also more likely to have their most recent physician appointment occur within the past six months ( $p=$ o.036). Analysis of matched-data from each age category supported a dependence between patient age and preferred information resource, as the physician was identified as responsible for a significant amount of counseling in the 26-34 year-old group ( $p=0.022)$.

In contrast, distribution of self-reported emotional reaction to the diagnosis of PCOS could not be predicted by any clinical parameter, and the feelings experienced 


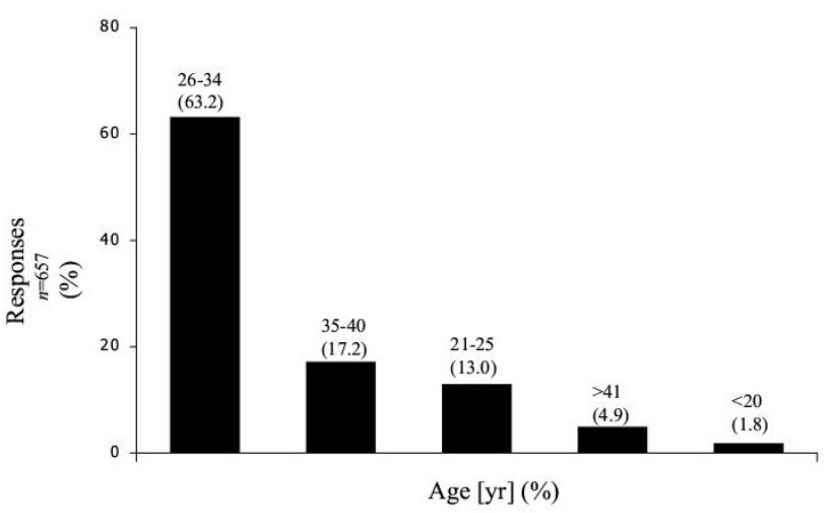

Figure I

Age distribution of polycystic ovary syndrome study participants.

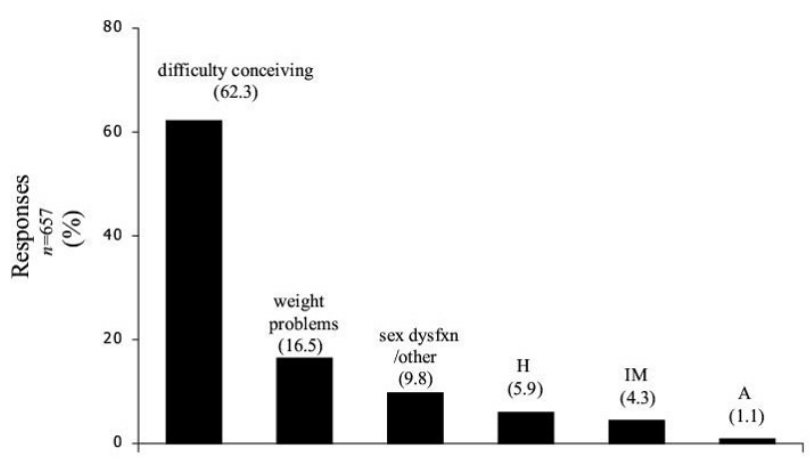

Figure 2

Most significant patient concern regarding polycystic ovary syndrome $(\mathrm{H}=$ hirsutism, $\mathrm{IM}=$ irregular menses, $\mathrm{A}=$ acne $)$

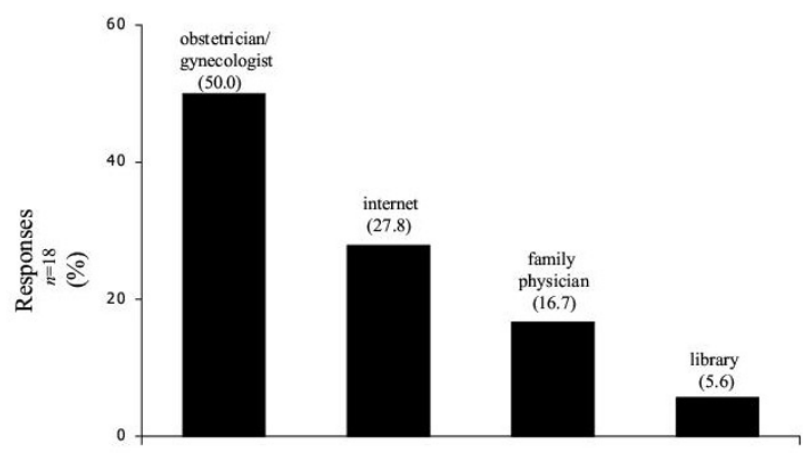

Figure 3

Educational resource most likely to be accessed first by respondents when additional information about PCOS is sought.

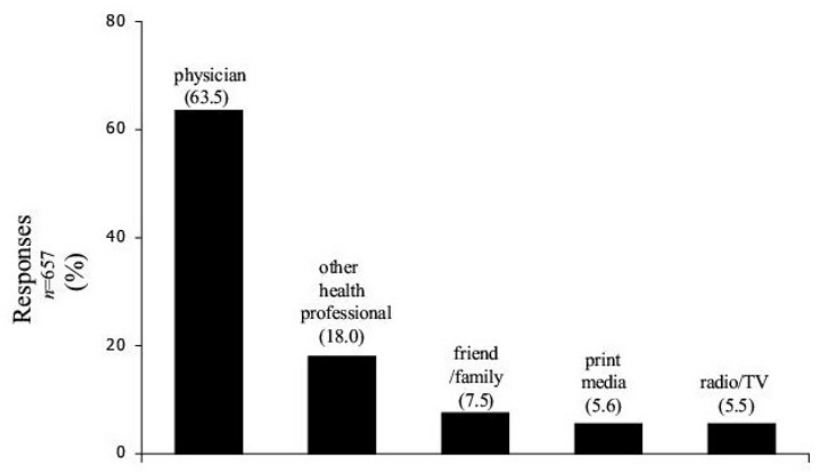

Figure 4

Initial source of information about PCOS among women with self-reported awareness of the disorder.

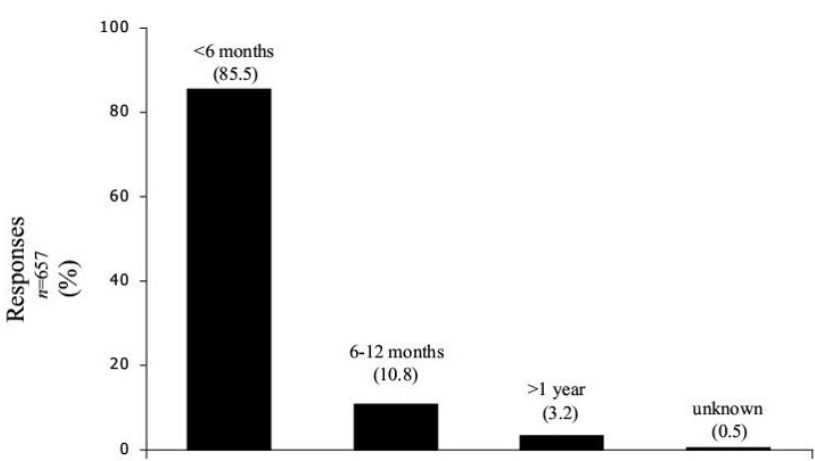

Figure 5

Time interval since most recent physician office visit.

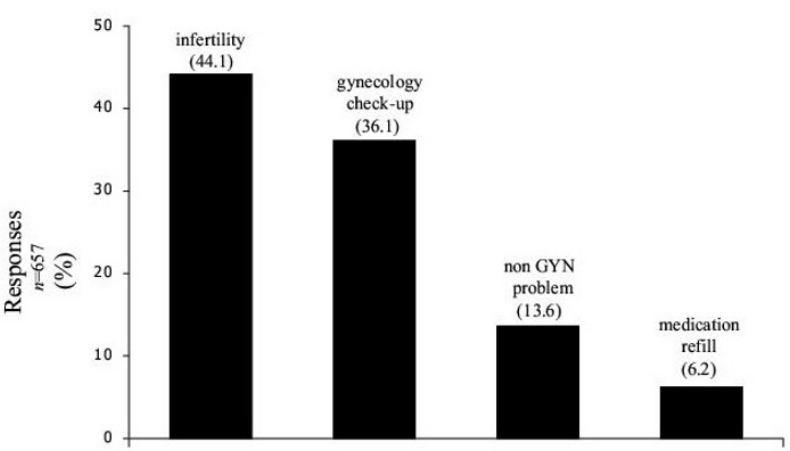

Figure 6

Self-reported reason for most recent appointment to see physician. 


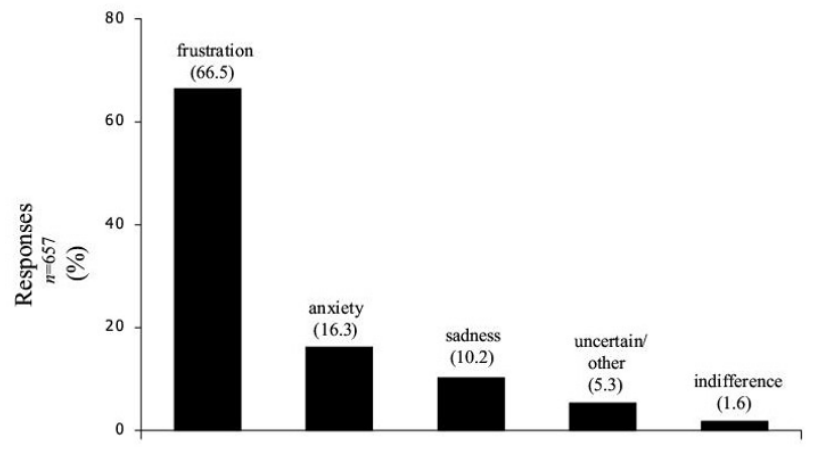

Figure 7

Patient emotions/feelings attributed to their diagnosis of PCOS.

about PCOS did not follow any age-specific pattern ( $p=$ o.268). There were, however, expected statistical dependencies between increasing age and ever-pregnant status $(p<0.001)$, as well as between age and history of receiving fertility therapy $(p<0.001)$. When asked: "If your PCOS could be safely and effectively helped by something else besides fertility drugs or birth control pills, would that interest you?", interest in alternative PCOS treatments was expressed by $99 \%$ of the sample ( $n$ $=648$ ). Assuming normal distribution of PCOS in the general population, power analysis defined the probability of detecting a significant $(p<0.05)$ relationship between dependent and independent variables at .80 with a sample of $n=657$.

The total number of page-views specifically attributed to the PCOS questionnaire site during the study period was 1,585 ( $41.5 \%$ response rate). Although completely voluntary, any prospective questionnaire viewer was given an opportunity to critique the instrument and post any concerns to the host. During the study there were no complaints or adverse outcomes regarding questionnaire content/format.

\section{Discussion}

Polycystic ovary syndrome (PCOS) describes a convergence of chronic multisystem endocrine derangements, including irregular menses, hirsutism, obesity, hyperlipidemia, androgenization, large and polycystic-appearing ovaries, insulin resistance and infertility [5]. Although PCOS is the most common hormonal disorder among reproductive age women [1], is the leading cause for anovulatory/oligoovulatory infertility [6], and has the potential for serious long-term health effects [7-10], paradoxically the condition is often underdiagnosed. The absence of uniform diagnostic criteria for PCOS [11] has probably contributed to its low clinical diagnosis rate, al- though organized media campaigns [12] aimed to increase PCOS awareness (both among physicians and patients) should result in more women seeking medical evaluation specifically because PCOS is suspected. As these laudable education efforts gain momentum, however, the current state of patient comfort with their "level of awareness" regarding PCOS, where their facts come from, and how they feel about their diagnosis are imprecisely characterized. If the objective of heightened public interest in PCOS is indeed achieved through wide publicservice announcements or other structured media exposure, then a contemporary (baseline) measurement of patient perception and awareness of PCOS as reported here would be helpful.

Although we could not verify the self-reported cognitive claims made by study participants, more than half of respondents considered themselves to be "very aware" of PCOS. These data confirm an intuitive association between physicians and PCOS patient teaching through office-based patient education and counseling. That most women would turn first to their doctor for further guidance regarding PCOS is not a new finding, although these data indicate that the messages conveyed by doctors appears to be poorly received by patients.

Specifically, the strongly negative emotions study participant identified with PCOS management by physicians demonstrated a profound gap between patient expectations and actual (prescribed) therapy. Indeed, our study population appeared unsatisfied with oral contraceptives or clomiphene citrate when prescribed as treatment for PCOS. In this study we were careful not to call specific attention to any substitute therapy, but rather to describe patient acceptance of these two existing treatments. This investigation detected a strong receptiveness among PCOS women regarding any safe and effective alternative to PCOS management. "Frustration" and "anxiety" associated with PCOS was observed across all age groups in this sample, perhaps suggesting either that a PCOS diagnosis was delayed in some older patients, or that negative emotions persist in some PCOS patients for many years after they are informed of their condition.

As with any questionnaire-based research, there were some important limitations with our study that should be acknowledged. Our research method relied on an unscreened but motivated audience and a functional computer interface, with the result that PCOS patients lacking the means and/or ability to access the study questionnaire were excluded from our sample. The fact that study participation depended on internet use likely explains the high self-reported familiarity and knowledge level for PCOS in this group. While PCOS preferen- 
tially affects women of reproductive age, and patients in this age group may tend to have a greater fluency with computer-based research tools, a full exploration of this demographic association was beyond the scope of our investigation. Conversely, the possibility also existed that some of our respondents had not been properly diagnosed with PCOS and therefore inappropriately inflated the sample. We regarded these two balanced sources of selection bias to be of essentially equivalent magnitude and therefore mutually negating. Whether or not the observations reported by computer-assisted questionnaire are representative of all PCOS patients is difficult to establish, although this represents the focus of ongoing research at our institutions. It may be that results obtained exclusively from an anonymous, confidential computeraccessed questionnaire are more likely to depict extreme views not typical of those encountered clinically, yet the sample size registered here was considered sufficiently large to attenuate this effect.

\section{Conclusion}

Efforts to heighten the profile of PCOS among the general public remain critical, as "minimal awareness" or "no awareness" of PCOS was present among $>10 \%$ of this motivated sample of unscreened patients. Although many women are affected by the polycystic ovary syndrome, difficulties associated with its uniform diagnosis persist. Given the diagnostic challenges of PCOS, there is perhaps little wonder that confusion also accompanies its treatment. Our work suggests that the present state of mainstream clinical management for this common endocrine disorder (birth control pills or clomiphene) appears to fall short of patient expectations, and therefore may represent a ready clinical niche for newer therapeutic modalities.

\section{Competing interests}

None declared.

\section{Acknowledgements}

The authors are grateful to the computational informatics division at OBGYN.net for their work in facilitating data collection for analysis. We are especially appreciative of the many women who participated in this questionnaire and made our research possible.

\section{References}

I. Cronin L, Guyatt G, Griffith L, et al: Development of a health related quality of life questionnaire for women with polycystic ovary syndrome (PCOS). J Clin Endocrinol Metab 1998, 83:1976-87

2. Kidson W: Polycystic ovary syndrome: a new direction in treatment. Med J Aust 1999, 169:537-40

3. Jakubowicz DJ, Seppala M, Jakubowicz S, et al: Insulin reduction with metformin increases luteal phase glycodelin and insulinlike growth factor-binding protein I concentrations and enhances uterine vascularity and blood flow in the polycystic ovary syndrome. J Clin Endocrinol Metab 200I, 86:1 126-33

4. Sills ES, Levy DP, Wittkowski KM, Perloe M: Preliminary experience with metformin treatment of polycystic ovary syndrome: reproductive outcomes among 20 consecutive patients. Fertil Steril 1999, 72(SuppI I):SI87-8
5. Sills ES, Perloe M, Palermo GD: Correction of hyperinsulinemia in oligoovulatory women with clomiphene-resistant polycystic ovary syndrome: a review of therapeutic rationale and reproductive outcomes. Eur J Obstet Gynecol Reprod Biol 2000, 9I:|35-4|

6. Hull MGR: Epidemiology of infertility and polycystic ovarian disease: endocrinological and demographic studies. Gynecol Endocrinol 1987, 1:235-45

7. Talbott EO, Guzick DS, Sutton-Tyrrell K, et al: Evidence for association between polycystic ovary syndrome and premature carotid atherosclerosis in middle-aged women. Arterioscler Thromb Vasc Biol 2000, 20:24I4-2I

8. Solomon CG, Hu FB, Willett WC, et al: History of irregular menstrual cycles and risk for coronary heart disease [abstract \#l 07920]. 73rd Annual Scientific Session, American Heart Association, 1999

9. Zborowski JV, Talbott EO, Canley JA: Polycystic ovary syndrome, androgen excess, and the impact on bone. Obstet Gynecol Clin North Am 200।, 28:135-5।

10. Kelly C), Connell JM, Cameron IT, Gould GW, Lyell H: The long term health consequences of polycystic ovary syndrome. $\mathrm{Br} J$ Obstet Gynaecol 2000, 107:1327-38

II. Hacihanefioglu B: Polycystic ovary syndrome nomenclature: chaos? Fertil Steril 2000, 73:1261-2

12. Jancin B: Targeting primary care providers: endocrine society aims to raise PCOS awareness. Ob. Gyn News 200037

\section{Pre-publication history}

The pre-publication history for this paper can be accessed here:

http://www.biomedcentral.com/content/backmatter/ 1472-6874-1-3-b1.pdf

Publish with BioMed Central and every scientist can read your work free of charge

"BioMedcentral will be the most significant development for disseminating the results of biomedical research in our lifetime." Paul Nurse, Director-General, Imperial Cancer Research Fund

Publish with BMC and your research papers will be:

- available free of charge to the entire biomedical community

- peer reviewed and published immediately upon acceptance

- cited in PubMed and archived on PubMed Central

- yours - you keep the copyright

Submit your manuscript here: BioMedcentral.com http://www.biomedcentral.com/manuscript/ editorial@biomedcentral.com 